Relations industrielles

Industrial Relations

\title{
International Labour Conventions and Recommandations, I.L.O., Geneva, 1982, 1167 pp.
}

\section{Alain Barré}

Volume 38, numéro 1, 1983

URI : https://id.erudit.org/iderudit/029343ar

DOI : https://doi.org/10.7202/029343ar

Aller au sommaire du numéro

Éditeur(s)

Département des relations industrielles de l'Université Laval

ISSN

0034-379X (imprimé)

1703-8138 (numérique)

Découvrir la revue

Citer ce compte rendu

Barré, A. (1983). Compte rendu de [International Labour Conventions and Recommandations, I.L.O., Geneva, 1982, 1167 pp.] Relations industrielles / Industrial Relations, 38(1), 189-190. https://doi.org/10.7202/029343ar

Tous droits réservés (C) Département des relations industrielles de l'Université Laval, 1983
Ce document est protégé par la loi sur le droit d'auteur. L’utilisation des services d'Érudit (y compris la reproduction) est assujettie à sa politique d'utilisation que vous pouvez consulter en ligne.

https://apropos.erudit.org/fr/usagers/politique-dutilisation/ 
the Board's enemies in Congress triumphed in the 1938 elections over FDR's attempt to purge them. The resulting coalition of Republicans and southern Democrats brought a halt to the New Deal and nearly scuttled the Wagner Act. Led by Howard Smith of Virginia, a special House of Representatives committee launched a ruthless investigation of the NLRB in 1939 that was supported by the AFL, business groups, and the Republican party. Smith supoenaed NLRB records and manipulated testimony to produce much unfavorable publicity for the NLRB. Denied access to their own records, Board members struggled to defend themselves. But their dry recitation of facts was no match for unuendos and allegations carefully timed to appear in the evening newspapers, and "the result was a one-sided and often distorted appraisal" (203) of the Board's work. Smith's bill to abolish the NLRB never became law. But Gross concludes that the hearings " triggered drastic and long-lasting changes in American labor policy" (225) by creating the climate for passage of the Taft-Hartley Act in 1947, a law which confirmed the near-fatal transformation of the NLRB from the vigorous independent agency its founders had intended into a much weaker sister.

The author blames the NLRB, Congress, and the AFL for undermining the Wagner Act. The Board should have been more sensitive to political pressures, but in my view he goes too far when he implies that civil servants should not have the same freedom as ordinary citizens to exercise their political rights (chapter 7). Gross criticizes Congress for reducing the effectiveness of the Board by abolishing its research staff. The author's heaviest ammunition is saved for the AFL, whom he accuses of acting irresponsibly and deceiving their own membership. AFL leaders, he says, were largely responsible for the "abhorrent" Taft-Hartley Act.

The book is a good read, but it is not a particularly easy one. The author's long, awkward sentences, his heavy reliance upon purely informational quotations, and his devotion to extraneous detail are often bothersome. Gross was not well served by a publisher who should have revised the manuscript to eliminate repetition in both content and writing style, corrected the numerous typographical errors, and improved the overall organization. Yet the author's arguments are persuasive and welldocumented, and the book excels at reconstructing the fascinating political ocean on which the NLRB foundered and nearly sank during its first decade.

If only because P.C. 1003 (1944) was modelled upon the Wagner Act, the book is worthwhile for Canadians to ponder. A recent article by Laurel MacDowell (Labour/ Le Travailleur 3, 1978) reveals that the outcome was the same despite the very different political context. Far from desiring to shore up the bargaining power of workers, the King government sought only to eliminate wartime industrial unrest. By wedding the compulsory conciliation features of Canada's old labour law to the Wagner Act model, P.C. 1003 anticipated Taft-Hartley's "cooling-off" provisions. Thus for both Canadians and Americans, the New Deal intervention into the historic struggle between employers and workers both legalized and politicized industrial relations.

Robert H. BABCOCK

University of Maine at Orono

International Labour Conventions and Recommandations, I.L.O., Geneva, 1982, $1167 \mathrm{pp}$.

Au terme d'une revue en profondeur des normes internationales du travail, le Conseil d'administration de l'O.I.T. décida en 1979 de la publication d'une nouvelle compilation des conventions et recommandations élaborées sous les auspices de l'O.I.T. Rappelons que le dernier ouvrage de ce genre remontait déjà à 1966: Conventions and Recommandations, I.L.O., Geneva, 1966. Depuis lors, de nombreux instruments furent adoptés par la 
Conférence internationale du travail. Cette nouvelle compilation ne constitue pas rigoureusement une seconde édition du recueil publié en 1966 puisque les conventions et recommandations ne sont plus présentées par ordre chronologique, mais bien par sujets.

L'ouvrage comporte deux tables des matières. La première suit l'ordre de représentation des instruments par sujets; la seconde, l'ordre chronologique. Ains, l'ouvrage pourra être aisément consulté tant par ceux qui cherchent à obtenir une information sur les normes de l'O.I.T. au regard d'un sujet donné, que par ceux qui, connaissant le numéro d'un instrument ou l'année de son adoption, désirent en consulter le texte.

Ce volume contient le texte de 146 conventions et 102 recommandations adoptées par la Conférence entre 1919 et 1981 . Les conventions qui ne figurent pas dans le recueil - dix au total - relèvent de trois catégories: les conventions qui n'ont pas reçu le nombre de ratifications nécessaire pour leur entrée en vigueur et qui ne sont plus ouvertes à la ratification à la suite de l'entrée en vigueur de conventions portant leur révision; les conventions qui n'ont pas encore reçu le nombre de ratifications nécessaire pour leur entrée en vigueur et qui furent révisées par une convention postérieure; les conventions portant révision des articles finals. Les soixante-trois recommandations non reproduites dans cette compilation ont été omises soit parce qu'elles ont été remplacées par une convention ou une recommandation postérieure, soit parce qu'elles sont de nature purement transitoire. Les différentes subdivisions de l'ouvrage comportent le cas échéant la liste des instruments non reproduits avec des renvois adéquats aux sources où les textes peuvent être consultés, soit au Compte rendu de la Conférence, soit au Bulletin officiel du B.I.T.

Les instruments intéressant plus d'un sujet sont reproduits dans la section la plus directement pertinente; la table des matières par sujets comporte à cet égard des renvois appropriés aux instruments publiés ailleurs dans le volume.
Enfin, signalons que seules les dispositions substantives des instruments sont reproduites. Les dispositions finales ainsi que les dispositions relatives aux déclarations d'application à des territoires non métropolitains sont reproduites dans deux appendices à la fin du volume. Sous chacune des conventions, des notes infrapaginales appropriées renvoient le cas échéant à ces appendices.

Compte tenu que la dernière compilation des instruments élaborés au sein de l'O.I.T. remontait déjà à 1966 , il n'est pas douteux que la publication de ce nouveau recueil sera saluée avec satisfaction par tous ceux qu'intéresse le développement du droit international du travail.

La version française de ce recueil sera publiée incessamment par le B.I.T.

Alain BARRÉ

Université Laval

Possono migliorare le relazioni industriali in Italia? par Gino Giugni, Luigi Mengoni, Felice Mortillaro et Dimitri Weiss, Torino, Gruppo Dirigenti Fiat, $1981,140 \mathrm{pp}$.

Le présent volume, dont le copyright est daté novembre 1981 mais qui a été, en réalité, publié en 1982, rassemble les actes de deux colloques successifs qui virent "face à face", respectivement en mai et en juin 1981, Felice Mortillaro, professeur chargé de cours à l'Université de Pérouse et directeur général de la Fédération patronale italienne de la métallurgie, Federmeccanica, et Gino Giugni, professeur titulaire de droit du travail à l'Université de Rome, co-auteur de la loi dite «Statut des travailleurs", pour le premier d'entre eux, et Dimitri Weiss, professeur titulaire de Sciences de gestion et relations industrielles à l'Université du Maine et à l'Institut d'Administration des Entreprises de l'Université de Paris I Panthéon-Sorbonne, et Luigi Mengoni, professeur titulaire de Droit civil à l'Université catholique de Milan, également co-auteur du Statut des travailleurs. 